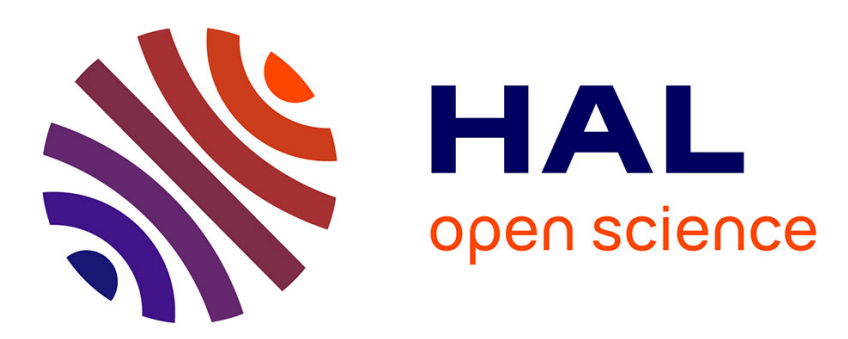

\title{
The melting behavior of organic materials confined in porous solids
}

\author{
Catheryn Jackson, Gregory B. Mckenna
}

\section{To cite this version:}

Catheryn Jackson, Gregory B. Mckenna. The melting behavior of organic materials confined in porous solids. Journal of Chemical Physics, 1990, 93 (12), pp.9002-9011. 10.1063/1.459240 . hal-02557807

\section{HAL Id: hal-02557807 \\ https://hal.science/hal-02557807}

Submitted on 29 Apr 2020

HAL is a multi-disciplinary open access archive for the deposit and dissemination of scientific research documents, whether they are published or not. The documents may come from teaching and research institutions in France or abroad, or from public or private research centers.
L'archive ouverte pluridisciplinaire HAL, est destinée au dépôt et à la diffusion de documents scientifiques de niveau recherche, publiés ou non, émanant des établissements d'enseignement et de recherche français ou étrangers, des laboratoires publics ou privés. 


\title{
The melting behavior of organic materials confined in porous solids
}

\author{
Catheryn L. Jackson and Gregory B. McKenna \\ The National Institute of Standards and Technology, Gaithersburg, Maryland 20899
}

(Received 6 July 1990; accepted 4 September 1990)

The solid-liquid phase transition temperatures and heats of fusion $\Delta H_{f}$ of nonpolar organic solids confined in the pores of controlled pore glasses were measured by differential scanning calorimetry. The pore diameters $d$ were in the range of $40-730 \AA$ and the organics studied were cis-decalin, trans-decalin, cyclohexane, benzene, chlorobenzene, naphthalene, and heptane. In accordance with previous reports on studies of primarily inorganic materials, the melting point of the pore solid $T(d)$ decreased with decreasing pore diameter. In addition, a large reduction in the bulk enthalpy of fusion $\Delta H_{f}$ of the pore solid was measured, which apparently has not been studied in detail by other workers. A linear correlation was found between the melting point depression $\left(\Delta T_{m}\right)$ and the reciprocal diameter, as predicted by theories of solidification in a capillary. The calculated values of the solid-liquid interfacial energy $\sigma_{\mathrm{sl}}$ were in reasonable agreement with values reported in the literature based on other methods of measurement.

\section{INTRODUCTION}

The behavior of liquids and solids in very small pores is relevant from both a fundamental and practical perspective. There is fundamental interest on the effect of finite size constraints on bulk properties. Crystalline melting, ${ }^{1-11}$ solid-solid ${ }^{5}$ and superfluid ${ }^{12}$ phase transitions are observed to shift to lower temperatures for materials confined to small pores. Also, the behavior of liquid in small pores has importance in many commercial areas such as catalysis, interfacial adhesion, and separation science. Water has been widely examined because of practical interest in frost prevention in biological systems, ${ }^{13}$ agricultural products, soil, ${ }^{14}$ and building materials. ${ }^{15}$

We are concerned with the crystalline melting temperature $T_{m}$ of organic solids formed in small pores of controlled pore glasses ${ }^{16}$ of varying pore diameter $d$. Although many earlier studies of the melting behavior of solids formed in small pores (pore solid) exist, the common feature is generally an estimate of $\Delta T_{m}$ at a single pore size, with no specific information of the effect of pore size on the melting behavior. In fact, very few studies of $T_{m}$ as a function of pore size exist, since narrow pore size distribution materials of a sufficiently small pore size were not readily available until the mid-1970's. A brief review of the literature will be given to place the present study in proper perspective.

The lowered melting temperature of pore solids has frequently been ascribed to a very small crystal size in the pore ${ }^{6,14}$ and the large surface to volume ratio of material adsorbed in a capillary ${ }^{14}$ The limitation of crystal size is known to lower the equilibrium melting point in other systems such as metal particles, ${ }^{17,18}$ finely dispersed nonporous powders, ${ }^{19}$ and polymer lamelle of different thicknesses. ${ }^{20}$ Very large effects have been reported for gold particles of $50 \AA$ size, which exhibit a $\Delta T_{m}$ of more than $500 \mathrm{~K} .{ }^{17}$ One expected and important feature of melting in small systems is the "smearing out" of the transition as the particle size decreases. This is due to the diffuse nature of the solid-liquid interface, which has been predicted by theory and shown by experiment for metal particles. ${ }^{21}$

The formation of small crystals is described theoretically in a classical discussion by Gibbs, ${ }^{22}$ and results from the effect of surface curvature on the equilibrium state of a pure substance. ${ }^{6}$ A related theory was derived by Thomson $^{23}$ for the effect of curvature on the vapor pressure of liquid droplets. This theory may be extended to small crystals to give the same equation as that of Gibbs, as noted by Defay et al. ${ }^{6}$ and the equation for the temperature shift of melting in confined geometries is often called the GibbsThomson equation. The Kelvin equation in its original form was found to correctly describe the facile condensation of a vapor in the pores of a solid, i.e., capillary condensation. ${ }^{7}$ A number of similar equations exist in the literature which have been derived for crystals formed in cylindrical pores ${ }^{3,8,14}$ which specifically consider the contact angle between the liquid, solid and the wall of the cylindrical pore, which is very important if freezing behavior is of interest. For crystal melting behavior, however, it is assumed that the contact angle is $180^{\circ}$ and the equation becomes identical with the Gibbs-Thomson equation, given below. We will reconsider the effect of the contact angle later in the Analysis section.

The Gibbs-Thomson equation predicts that the melting point depression $\Delta T_{m}$ for a small crystal of size $d$ is given by

$$
\Delta T_{m}=T_{m}-T_{m}(d)=4 \sigma_{\mathrm{sl}} T_{m} /\left(d \Delta H_{f} \rho_{s}\right),
$$

where $\sigma_{\mathrm{sl}}$ is the surface energy of the solid-liquid interface, $T_{m}$ is the normal (bulk) melting point, $T_{m}(d)$ is the melting point of crystals of size $d, \Delta H_{f}$ is the bulk enthalpy of fusion (per $\mathrm{g}$ of material), and $\rho_{s}$ is the density of the solid. Presented in this form, it is assumed that $\sigma_{\mathrm{sl}}$ is isotropic and that the crystal size is sufficiently large that the material retains its bulk properties for $\Delta H_{f}$ and $\rho_{s}$. The possible failure of these assumptions is considered in the Analysis section. 
Experimental reports of the depression of the bulk melting temperature of solids confined to a small system size are widespread, but many questions remain unanswered. For example, early calorimetric measurements on water adsorbed in porous silica gel, ${ }^{2}$ showed a broad melting range of $50 \mathrm{~K}$ and a reduction in the peak melting point of nearly $12 \mathrm{~K}$. For benzene and napthalene in the same system, however, an even larger reduction in the peak melting point $(\sim 40 \mathrm{~K})$ was found and the differences could not be adequately explained by the authors. ${ }^{2}$ In general, low molar mass inorganic liquids such as water, ${ }^{2,4,9-11}$ hydrogen, ${ }^{8}$ neon, ${ }^{8}$ and oxygen, ${ }^{3,5}$ have been studied more frequently than organic liquids. ${ }^{2,10}$

Another effect which is not well understood is the relationship between small system size and the enthalpy of fusion $\Delta H_{f}$ of the solid formed in the pores. Conflicting results on measurements made for $\Delta H_{f}$ of water in silica gel and other porous media have been noted, with $\Delta H_{f}$ reported to be reduced, ${ }^{4}$ increased, ${ }^{2}$ or unchanged ${ }^{11}$ for the pore solid. The hysteresis between freezing and melting in the liquid-solid transition of liquids confined in porous media has been a topic of study because of the large degree of supercooling which occurs in liquids such as hydrogen, ${ }^{8}$ where no significant supercooling occurs in the bulk.

Part of the diversity in reports of the melting behavior may be due to the different nature of the many porous "host" materials employed in the studies, including silica gels, ${ }^{2}$ porous sol-gel glasses, ${ }^{3,5}$ porous Vycor ${ }^{24}$ glass, ${ }^{4,8-10}$ and controlled pore glasses (CPGs). ${ }^{11}$ The different nature is manifested in the shape and breadth of the pore size distribution, surface roughness, and other features of these materials. One of the earliest materials examined was silica gel, made in a sol-gel process from a suspension of colloidal particles. The sol-gel process usually results in a very broad pore size distribution, although recent developments in solgel technology provide better control of the pore geometry, yielding samples with well-defined pore diameters. ${ }^{3}$ These materials are called "porous sol-gel glasses." Vycor brand porous glass (Corning Glass ${ }^{24} \# 7930$, pore diameter $\sim 50$ $\AA$ ) and controlled pore glasses (CPG Incorporated ${ }^{24}$ ) are produced by a phase separation process which is fundamentally different from the sol-gel process. ${ }^{16}$ CPGs have the advantage of being available in a number of different pore diameters. The morphology of these materials is a network of cylindrical pores, rather than isolated pores with one end closed. This allows the assumption that the contact angle remains constant within the pore and minimizes changes in $\Delta T_{m}$ as the pore is filled. The pore size distributions of sol-gel glasses, ${ }^{3}$ porous Vycor glass and controlled pore glasses ${ }^{16}$ are quite narrow compared to silica gels, which simplifies the interpretation of the experimental data.

The availability of CPGs and sol-gel glasses of different pore diameters has led to recent studies which give attention to the melting behavior as a function of pore size. Rennie and Clifford ${ }^{11}$ conducted a calorimetric study of water in CPG with pore diameters from $20-2500 \AA$. The results indicated that beyond a few monolayers, water exists essentially as bulk liquid in the pores and that the melting of pore ice was well described by the GibbsThomson equation. The authors also observed a direct proportionality between the amount of water determined calorimetrically and the total water present, indicating a negligible deviation in the heat of melting due to surface effects. This is in contrast to the earlier report of Litvan ${ }^{4}$ that $\Delta \mathrm{H}_{f}$ for water was significantly reduced in porous $V y c o r$ glass and contrary to our finding for organic liquids as will be seen subsequently. In addition, Rennie and Clifford $^{11}$ calculated experimental values for $\sigma_{\mathrm{sl}}$ of water at each pore diameter using Eq. (1), and concluded that it was not a function of diameter. Another study ${ }^{3}$ utilized a picosecond optical technique to study molecular dynamics of liquid oxygen in porous sol-gel glasses having pore diameters which ranged from $40-400 \AA$. From these experiments a $1 / d$ dependence of $\Delta T_{m}$ was also observed, however, no calorimetric data were available to investigate the enthalpy of fusion of the pore solid.

The present study investigates the melting behavior of a variety of organic solids in CPGs as a function of pore diameter. The melting point depression $\Delta T_{m}$ and the enthalpy of fusion $\Delta H_{f}$ were measured by differential scanning calorimetry (DSC). The CPG surfaces were derivatized to promote wetting by nonpolar, organic liquids. The organic liquids studied in detail were cis-decalin, transdecalin, cyclohexane, benzene, chlorobenzene, naphthalene and heptane. Most of the data were obtained with a slight excess loading of the pores. Under this condition, the bulk melting temperature and the lower pore solid melting temperature were both observed, as previously reported for water, benzene, and naphthalene in silica gel, ${ }^{2}$ and water in CPG. ${ }^{11}$ This condition has the added feature of minimizing the effect of surface forces on the pore material, therefore emphasizing the small size and isolation of the organic crystal. The data were analyzed according to the prediction of a linear relationship between $\Delta T_{m}$ and $1 / d$ given by Eq. (1). Values for $\sigma_{\mathrm{sl}}$ were calculated for each material and compared to empirical calculations and literature values based on other techniques, when available.

\section{EXPERIMENTAL}

\section{Materials}

The controlled pore glasses (CPG) used in this study were supplied by Dr. Wolfgang Haller of NIST. ${ }^{16,25}$ These materials are also available commercially from CPG Inc. in Fairfield, New Jersey. The CPG is a white, free-flowing powder of $120 / 200$ mesh size. The mean pore diameter, the range of diameters which describes $90 \%$ of the pores, and the pore volume for each CPG were determined by mercury intrusion and are given in Table $\mathrm{I}$. The surface area of the glass is also given in Table $I$, as determined by nitrogen adsorption using the Branauer-Emmett-Teller (BET) equation.

The surface of the CPG was derivatized with hexamethyldisilazane to convert the surface hydroxyl groups to trimethylsilyl groups. This treatment makes the glass more hydrophobic and promotes wetting by organic liquids. Each CPG was first cleaned by boiling in nitric acid on a 
TABLE I. Controlled pore glass properties.

\begin{tabular}{|c|c|c|c|}
\hline $\begin{array}{l}\text { Mean pore } \\
\text { diameter }{ }^{\mathrm{a}},(\AA)\end{array}$ & $\pm \%^{\mathrm{b}}$ & Surface area ${ }^{c}\left(m^{2} / g\right)$ & Pore volume ${ }^{\mathrm{a}}(\mathrm{cc} / \mathrm{g})$ \\
\hline 39.5 & 12.8 & 144 & 0.13 \\
\hline 85 & 13.5 & 175 & 0.59 \\
\hline 156 & 5.7 & 166 & 0.90 \\
\hline 255 & 3.7 & 95.5 & 0.96 \\
\hline 324 & 4.2 & 79.0 & 1.39 \\
\hline 486 & 3.9 & 43.3 & 0.92 \\
\hline 729 & 8.6 & 33.9 & 0.98 \\
\hline
\end{tabular}

anetermined by mercury intrusion method.

${ }^{6} 90 \%$ of the pore diameters are within this range.

'Determined by nitrogen adsorption method (BET equation).

steam bath for $10 \mathrm{~h}$. The nitric acid was then decanted off and the CPG was rinsed copiously with distilled, deionized water, and dried thoroughly in an oven. The hexamethyldisilazane was then added to cover the glass, stirred with a spatula to release trapped air bubbles, and heated at 50$55^{\circ} \mathrm{C}$ for $20 \mathrm{~h}$. Following this treatment, the reagent was decanted off and the CPG was rinsed well with chloroform and dried thoroughly in an oven. The CPGs were stored in a desiccator when not in use. It should be noted that the surface derivatization does not significantly effect the pore dimensions. ${ }^{26}$

The organic solvents used in this study were obtained from the following manufacturers ${ }^{24}$ and used without further purification: cis-decalin, trans-decalin, and hexadecane from Aldrich Chemical Company, $n$-heptane, cyclohexane, and naphthalene from Mallinckrodt, benzene from J. T. Baker, and chlorobenzene from Fisher Scientific.

\section{Calorimetry}

The Perkin-Elmer ${ }^{24}$ DSC $2 \mathrm{C}$ used in this study was specially modified for low temperature work. The modification involved building a new heat sink attachment for the DSC head to allow a continuous flow of liquid nitrogen through the system. This keeps the block at a constant low temperature with minimal operator attention, and improves the stability of the baseline and calibration. The attachment was made from a cylindrical piece of brass (7.5 $\mathrm{cm}$ in diameter and $3.0 \mathrm{~cm}$ in height $)$ with a $1.27 \mathrm{~cm}(1 / 2$ in.) hole in the center to bolt it to the DSC head in the usual manner. The brass piece was machined out to make two cylindrical chambers (around the center hole) separated by a thin sheet of brass. A hole was then drilled into the side of each chamber and fit with a $0.64 \mathrm{~cm}(1 / 4 \mathrm{in}$.) fitting to pipe the liquid nitrogen in and out of the attachment. Flow between the chambers was controlled with a $0.32 \mathrm{~cm}(1 / 8 \mathrm{in}$.) hole. The open end of each chamber was sealed with a piece of brass. The lowest starting temperature achievable with this attachment was $\sim 140 \mathrm{~K}$.

The DSC sample pans used in this study were large volume, stainless-steel pans sealed with an O-ring to prevent evaporation of the solvent (Perkin-Elmer \#03190218). The method of sample preparation was to weigh the CPG into the preweighed sample pan, add the liquid (or solid in the case of naphthalene), seal the pan, and reweigh. The precision of the balance was $\pm 0.01 \mathrm{mg}$. The concentrations reported in $\mathrm{cm}^{3} / \mathrm{g}$ are in $\mathrm{cm}^{3}$ of liquid, calculated using the liquid densities, ${ }^{27}$ per $\mathrm{g}$ of glass. The CPG/liquid mixtures were equilibrated overnight at room temperature while the solid solvents were heated to $\sim 90^{\circ} \mathrm{C}$ for a few hours to liquify the material so that it could flow into the pores. Before analysis the samples were reweighed to insure that the pans were sealed properly. In most cases, the reweighed samples were within $\pm 0.05 \mathrm{mg}$. A few pans showed a large weight loss due to improper sealing and were discarded. The prepared sample pans were stable over many months for all of the mixtures except CPG/benzene, which showed a small weight loss with time due to diffusion of the benzene through the rubber $O$ ring. The CPG/benzene mixtures were always weighed both before and after the sample run to assure that the weight was correct. No calorimetric effects due to the $O$ ring were observed.

Calibration of the DSC was done with pure samples of the organic liquids. The heating rate used was $5 \mathrm{~K} / \mathrm{min}$ and most of the data discussed in this paper was obtained on heating. A cooling rate of $5 \mathrm{~K} / \mathrm{min}$ was used to prepare each sample for analysis. The normal melting point and enthalpy of fusion for each solvent determined by DSC, as well as the literature values, are given in Table II. At the relatively low heating rate used in this study, good agreement was observed between the experimental peak melting points and the literature melting points. The experimentally measured values of bulk $\Delta H_{f}$ are also listed in Table II

TABLE II. DSC calibration.

\begin{tabular}{|c|c|c|c|c|c|c|}
\hline \multirow[b]{2}{*}{ Substance } & \multicolumn{3}{|c|}{ Literature values } & \multicolumn{3}{|c|}{ Experimental (peak) values } \\
\hline & $T_{m}(\mathrm{~K})$ & $\Delta H_{f}(\mathrm{cal} / \mathrm{g})$ & Source (Ref.) & $T_{m}(\mathrm{~K})$ & $\Delta H_{f}(\mathrm{cal} / \mathrm{g})$ & $T_{m}{ }^{\mathrm{a}}(\mathrm{K})$ \\
\hline n-heptane & 182.4 & 33.78 & 27 & 180.2 & 30.2 & 181.0 \\
\hline Chlorobenzene & 227.8 & 20.40 & 27 & 229.0 & 19.7 & 229.1 \\
\hline$c$-decalin & 230.2 & 16.4 & 28 & 230.1 & 15.7 & 229.9 \\
\hline$t$-decalin & 242.6 & 24.9 & 28 & 241.7 & 23.2 & 243.5 \\
\hline n-decane & 243.3 & 48.34 & 27 & 244.1 & 47.3 & 244.7 \\
\hline Benzene & 278.5 & 30.45 & 27 & 279.0 & 29.3 & 280.8 \\
\hline Cyclohexane & 279.5 & 7.47 & 27 & & & 280.1 \\
\hline$o$-terphenyl & 329.3 & 17.84 & 29 & 331.3 & 17.5 & 331.0 \\
\hline Naphthalene & 353.4 & 35.06 & 27 & 357.0 & 34.9 & 356.1 \\
\hline
\end{tabular}

${ }^{a}$ Average value for bulk melting peak in CPG/liquid mixtures where pore and bulk melting peaks are clearly resolved. 
and agree satisfactorily with the literature values. In all cases, the experimentally measured value of the bulk $\Delta H_{f}$ was used for calculations involving the pore melting peak enthalpy. The final column in Table II lists the average peak melting temperature of the excess bulk solvent in CPG/solvent samples where the pore and bulk melting peaks were clearly resolved. Because of the presence of the CPG, this peak tended to be slightly broader than that of the pure solvent, but in general was still in good agreement with the expected melting temperature.

A minimum temperature range of $100 \mathrm{~K}$ was studied for each system, from 80 below to $20 \mathrm{~K}$ above the normal melting point. The exceptions were the low melting materials $n$-heptane and chlorobenzene, where a slightly smaller range had to be used because the minimum temperature attainable with the instrument was $140 \mathrm{~K}$. In some cases a larger range was studied and no significant differences in the results were observed. Annealing of several of the samples for $3-4 \mathrm{~h}$ at low temperatures also did not significantly alter the observed melting temperature, the shape of the melting peak or $\Delta H_{f}$

The melting point depression $\Delta T_{m}$ was calculated in two different ways, depending on the resolution of the pore and bulk melting peaks. In the CPGs of smallest pore size, usually 40,85 , and $156 \AA$, the two peaks were well resolved and a direct measurement of $\Delta T_{m}$ was possible for each case. In the CPGs of larger pore size, the value of the bulk melting peak was taken as the last column in Table II. The broadened peak melting temperature (or a clearly resolved left-hand shoulder if visible) was then subtracted from this bulk melting temperature to obtain $\Delta T_{m}$.

\section{RESULTS AND ANALYSIS}

\section{Effect of pore fullness}

The effect of pore fullness on $\Delta T_{m}$ and $\Delta H_{f}$ was examined for both cis-decalin and benzene. The $85 \AA$ CPG was used for these studies, which clearly shows the separation between the pore-melting and bulk-melting temperatures, and allows an accurate measurement of $\Delta H_{f}$. Typical normalized data for cis-decalin is shown in Fig. 1 for concentrations of $0.133-0.998 \mathrm{~cm}^{3} / \mathrm{g}$. The peak melting temperatures of the pore solid and corresponding $\Delta H_{f}$ are given in Table III. At concentrations of $0.630 \mathrm{~cm}^{3} / \mathrm{g}$ and below, a single melting endotherm was observed at a peak melting temperature of $212-213 \mathrm{~K}$, about $17-18 \mathrm{~K}$ below the normal melting point $(230 \mathrm{~K})$. At concentrations of $\geqslant 0.718$ $\mathrm{cm}^{3} / \mathrm{g}$ and above, the bulk melting peak appeared at the normal melting point for cis-decalin, which is due to excess material which cannot fit in the pores. The expected pore volume for the $85 \AA$ CPG (obtained by mercury intrusion data given in Table I) is $0.59 \mathrm{~cm}^{3} / \mathrm{g}$, slightly lower than that observed experimentally for cis-decalin. This may be a result of different wetting characteristics of the two liquids on the glass surface. A small increase of $\sim 1 \mathrm{~K}$ in $T_{m}(d)$ for $c i s$-decalin is seen when the pores are completely filled, and the precision in the measurement is improved, as listed in Table III.

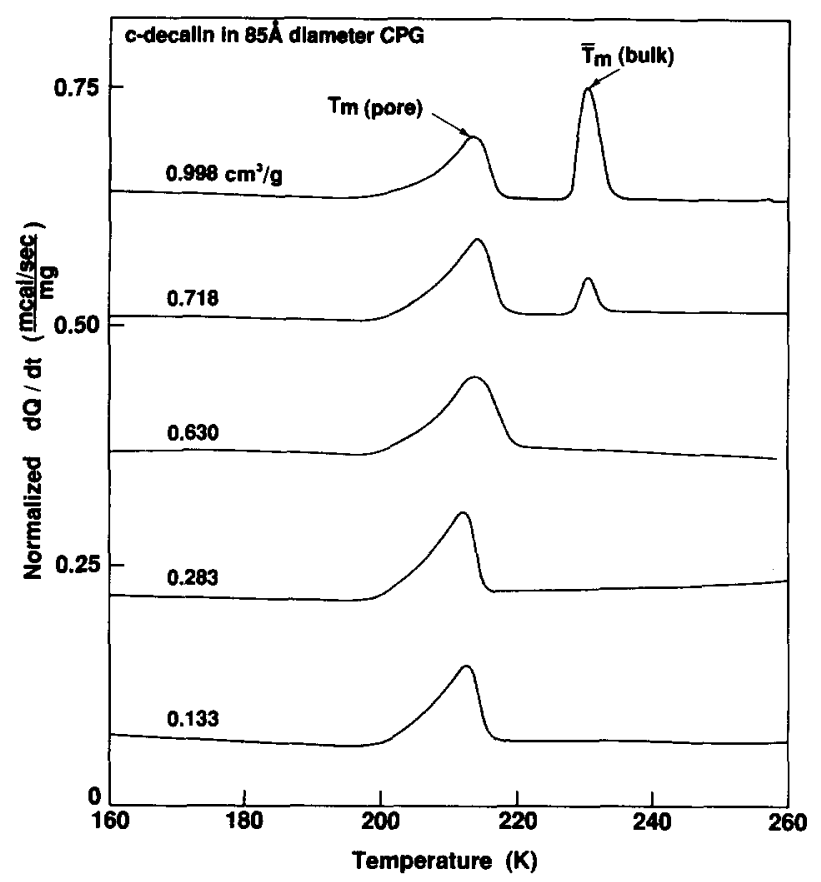

FIG. 1. Effect of pore fullness on the melting behavior: DSC heating curves of $c$-decalin in $85 \AA$ pores of CPG (heating rate $=5 \mathrm{~K} / \mathrm{min}$ ). At $0.133-0.630 \mathrm{~cm}^{3} / \mathrm{g}$, the pores are underfilled and only the pore solid melting endotherm appears, while at $0.718-0.998 \mathrm{~cm}^{3} / \mathrm{g}$ the pores are overfilled and a second melting endotherm appears due to the excess pore bulk solid. See text for further analysis.

Within the error of this measurement, the shape, melting temperature, and enthalpy of the pore melting peak shown in Fig. 1 for cis-decalin is independent of pore fullness. This suggests that the liquid prefers to condense as a plug in the center of a few pores rather than to wet the entire inside surface of the CPG uniformly, and that the glass surface is not playing a highly interactive role in these studies. If a uniform wetting of the interior surface were occurring, our results would more closely resemble the earlier study of water in untreated CPG by Rennie and Clifford, ${ }^{11}$ in which water was undetectable at $<15 \mathrm{wt}$. \% because of the presence of a few monolayers coating the entire surface before any pores were full. Cis-decalin was easily detected in the $85 \AA$ CPG as low as $0.067 \mathrm{~cm}^{3} / \mathrm{g}$, which corresponds to $5.7 \mathrm{wt}$. $\%$. Since $\Delta H_{f}$ of $c i s$-decalin is about one-fifth that of water, this represents a very great difference in the detection limit. The breadth of the pore melting endotherms shown in Fig. 1 are approximately 15 $\mathrm{K}$, with the peak shape somewhat skewed toward higher temperature. The peak shape and breadth is indicative of the pore size distribution as discussed previously by Homshaw, ${ }^{30}$ for water saturated ion-exchange resins and clays, and Brun et al. ${ }^{31,32}$ with regard to thermoporometry.

Similar results on the effect of pore fullness were found for benzene in the $85 \AA \mathrm{CPG}$, shown in Fig. 2 for concentrations of $0.15-0.923 \mathrm{~cm}^{3} / \mathrm{g}$. In this case, $\Delta T_{m}$ is $\sim 14-15$ $\mathrm{K}$. The shape of the pore melting peak for benzene at low pore filling $\left(0.150-0.553 \mathrm{~cm}^{3} / \mathrm{g}\right)$ differs from that observed for cis-decalin, with a small shoulder on the right-hand side (high temperature side) of the peak. A bulk melting peak 
TABLE III. Melting behavior of pore solid as a function of pore fullness in $85 \AA$ diameter CPG.

\begin{tabular}{|c|c|c|c|c|c|}
\hline Substance & $\begin{array}{l}\text { Experimental } \\
\text { condition }\end{array}$ & $(\mathrm{cc} / \mathrm{g})$ & & $T_{m}(d)^{\mathrm{a}}(\mathrm{K})$ & $\Delta H_{f}(\mathrm{cal} / \mathrm{g})$ \\
\hline \multirow[t]{11}{*}{$c$-decalin } & & 0.067 & & 211.2 & 5.8 \\
\hline & & 0.133 & & 212.2 & 8.0 \\
\hline & Underfilled & 0.283 & & 211.6 & 8.1 \\
\hline & pores & 0.373 & & 212.1 & 8.1 \\
\hline & & 0.630 & & 213.3 & 8.8 \\
\hline & & & avg. ${ }^{b}$ & 212.3 & 8.3 \\
\hline & & & $\sigma^{\circ}$ & 0.7 & 0.4 \\
\hline & Overfilled & 0.718 & & 213.4 & 8.6 \\
\hline & pores & 0.998 & & 212.8 & 9.0 \\
\hline & & & avg. ${ }^{b}$ & 213.1 & 8.8 \\
\hline & & & $\sigma^{\mathcal{C}}$ & 0.4 & 0.3 \\
\hline \multirow[t]{13}{*}{ Benzene } & & 0.066 & & 263.4 & 14.7 \\
\hline & Underfilled & 0.150 & & 266.7 & 18.6 \\
\hline & pores & 0.334 & & 264.7 & 19.9 \\
\hline & & 0.553 & & 263.8 & 19.4 \\
\hline & & & avg. ${ }^{b}$ & 265.1 & 19.3 \\
\hline & & & $\sigma^{\varepsilon}$ & 1.5 & 0.7 \\
\hline & & 0.704 & & 267.0 & 19.0 \\
\hline & Overfilled & 0.791 & & 266.0 & 21.1 \\
\hline & pores & 0.923 & & 266.6 & 19.7 \\
\hline & & 1.56 & & 266.6 & 20.0 \\
\hline & & 1.76 & & 266.1 & 19.5 \\
\hline & & & avg. ${ }^{b}$ & 266.5 & 19.9 \\
\hline & & & $\sigma^{2}$ & 0.4 & 0.8 \\
\hline
\end{tabular}

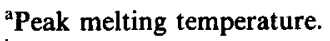

${ }^{\mathrm{b}}$ Avg. = mean of data, for underfilled pores the lowest $\mathrm{cc} / \mathrm{g}$ data is not included.

${ }^{\mathrm{c}} \sigma=$ one standard deviation.

also appears at lower pore filling, perhaps due to the higher vapor pressure of benzene compared to cis-decalin and the possible transport of some of the pore liquid out of the CPG. The shoulder disappears when the pores are filled to slight excess $\left(0.704-0.923 \mathrm{~cm}^{3} / \mathrm{g}\right)$. As noted previously for cis-decalin, a small increase of $\sim 1 \mathrm{~K}$ in $T_{m}(d)$ is measured with the pores completely filled, as compared to partially filled, and the precision in the measurement of $T_{m}(d)$ is increased (Table III). The breadth of the pore melting endotherm is similar for both compounds.

Although $\Delta H_{f}$ of the pore solid is fairly independent of pore fullness $\left(>0.13 \mathrm{~cm}^{3} / \mathrm{g}\right)$ for both cis-decalin and benzene, it is greatly reduced compared to the bulk $\Delta H_{f}$, as shown in Table III. For cis-decalin, the value of $\Delta H_{f}$ for the pore solid is $\sim 8-9 \mathrm{cal} / \mathrm{g}$ compared to a bulk $\Delta H_{f}$ of $15.7 \mathrm{cal} / \mathrm{g}$. For benzene, the reduction is slightly less but still significant. At the lowest pore filling, used to find the detection limit of the technique, an additional reduction in $\Delta H_{f}$ was measured. This may be caused in part by the error in measuring such a small peak. The reduction in $\Delta H_{f}$ of pore solid was found to vary with pore diameter (or crystal size) and will be considered in detail later.

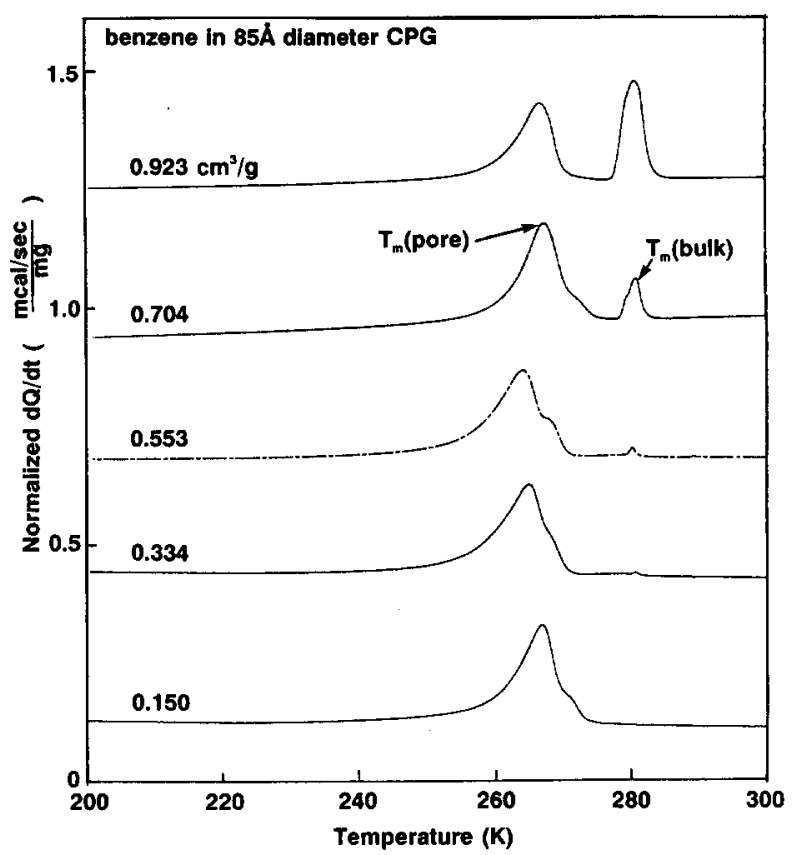

FIG. 2. Effect of pore fullness on the melting behavior: DSC heating curves of benzene in $85 \AA$ pores of CPG (heating rate $=5 \mathrm{~K} / \mathrm{min}$ ). At $0.150-0.553 \mathrm{~cm}^{3} / \mathrm{g}$ the pores are underfilled and at $0.704-0.923 \mathrm{~cm}^{3} / \mathrm{g}$ the pores are filled to excess, as described in Fig. 1.

\section{Effect of pore diameter on $\Delta T_{m}$}

Studies of the effect of pore fullness allowed us to establish appropriate conditions to study the effect of pore diameter on $\Delta T_{m}$ for a number of different organic materials. Because a slight excess filling of the pores provides an internal standard for the bulk melting temperature, we chose this condition to obtain most of the data described here. Direct measurement of $\Delta T_{m}$ is then possible when a large enough separation of the pore and bulk melting peaks occurs. One set of experimental DSC data will be given for benzene as a function of pore diameter to illustrate $\Delta T_{m}$ and $\Delta H_{f}$, similar data obtained for the remaining compounds will then be summarized graphically.

The effect of CPG pore diameter on the melting temperature of the benzene frozen in the pores is shown in Fig. 3. In the $40 \AA$ pores, $\Delta T_{m}$ is $\sim 26-27 \mathrm{~K}$, based on the peak melting temperatures, and the breadth of the peak is $\sim 45$ $K$. The broader nature of this peak may be partially due to the broader CPG pore size distribution at the $40 \AA$ size (Table I) and to the smearing effect of the liquid-solid transition at small particle size. ${ }^{21}$ The pore solid melting endotherm moves to higher temperatures as the pore diameter is increased to 85 and $156 \AA$, and eventually merges with the bulk (excess pore) melting peak. A typical DSC trace for pure, bulk benzene is shown at the bottom of Fig. 3 for reference.

Similar calorimetric data were obtained for cis-decalin, trans-decalin, cyclohexane, chlorobenzene, naphthalene, and heptane in the investigation of the effect of molecular size on $\Delta T_{m}$ and $\Delta H_{f}$. The results for $\Delta T_{m}$ are summarized in Fig. 4 , where $\Delta T_{m}$ is plotted versus $1 / d$. In general, a linear relationship is observed, in qualitative agreement 


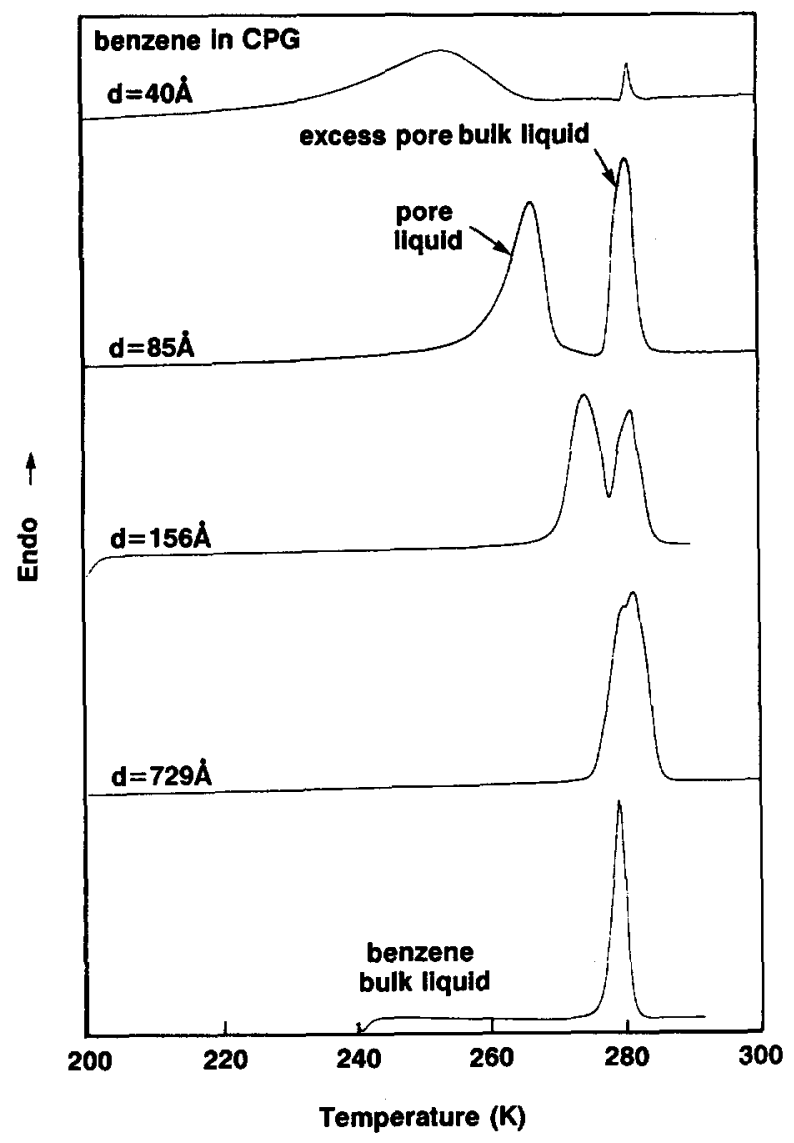

FIG. 3. Effect of pore diameter on the melting behavior: DSC heating curves of benzene in pore diameters of $40,85,156$ and $729 \AA$ CPG and compared to pure benzene standard (heating rate $5 \mathrm{~K} / \mathrm{min}$ ). The pores are filled to slight excess to show $\Delta T_{m}$ directly.

with Eq. (1). These results and the evaluation of $\sigma_{\mathrm{sl}}$ will be discussed in the Analysis section.

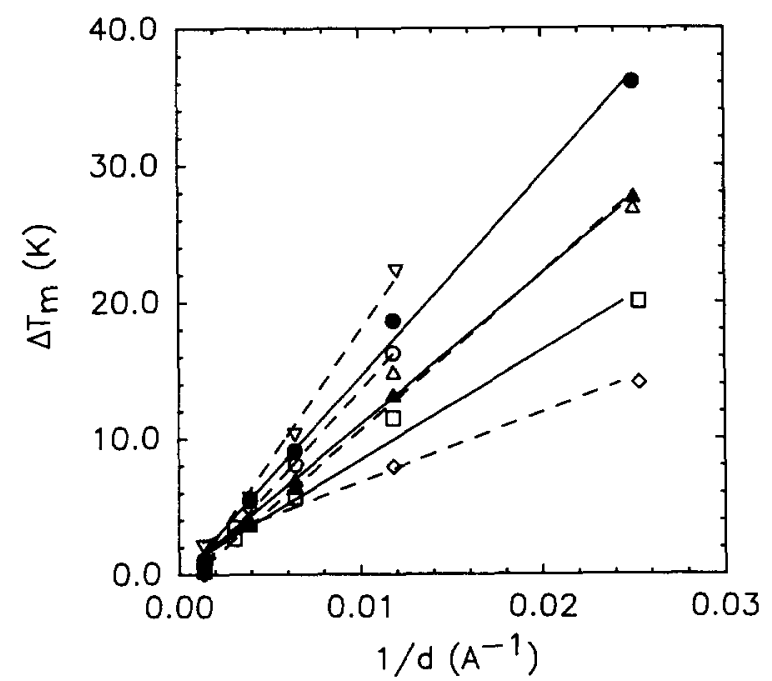

FIG. 4. Experimental values of $\Delta T_{m}$ plotted as a function of the reciprocal pore diameter for $(O)$ cis-decalin, $(\Theta)$ trans-decalin, $(\nabla)$ cyclohexane, $(\triangle)$ benzene, $(\Delta)$ chlorobenzene, $(\Delta)$ naphthalene and $(\square)$ heptane. The lines through the data are linear regressions fits used to calculate values of $\sigma_{\mathrm{s} 1}$. Melting endotherms were not observed in the smallest pores $(40 \AA)$ for cyclohexane and cis-decalin, see text for details.

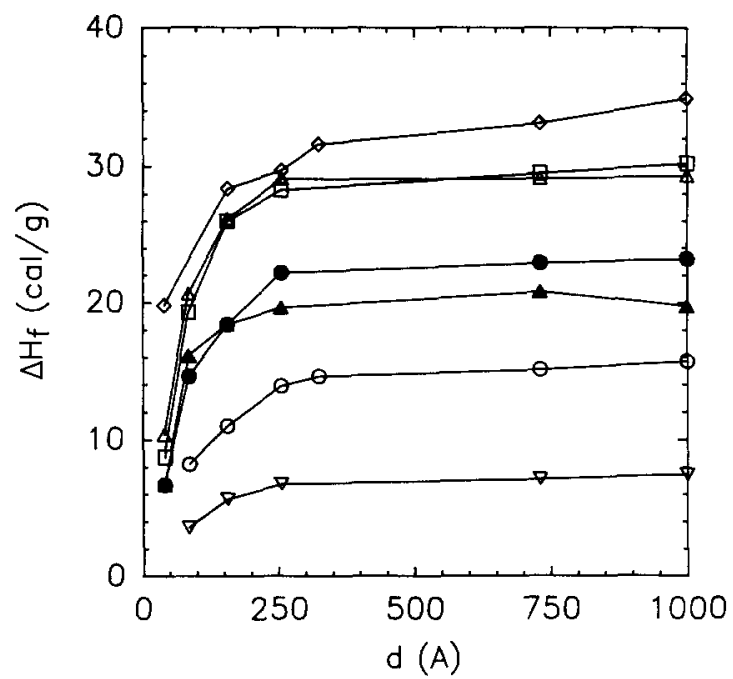

FIG. 5. Experimental values of $\Delta H_{f}$ of the pore solid vs $d$, showing a large reduction in the bulk $\Delta H_{f}$ (plotted at $1000 \AA$ for reference) in the smallest pores. Symbols are the same as given in Fig. 4 caption.

In the case of the cis-decalin and cyclohexane in the smallest diameter pores $(40 \AA)$, no melting endotherm was detected down to $145 \mathrm{~K} \cdot{ }^{33}$ Assuming the $1 / d$ dependence of $\Delta T_{m}$ (Fig. 4) to be valid, $T_{m}$ at $40 \AA$ is expected at about $205 \mathrm{~K}$ for $c$-decalin and $233 \mathrm{~K}$ for cyclohexane, well within the range of study. The lack of a melting endotherm may be due to the rather low bulk $\Delta H_{f}$ of these compounds compared to the other organics studied (Table II) in combination with the reduction of the bulk $\Delta H_{f}$ observed in the smallest pores, to be discussed in the next section. The possibility that these two liquids might not flow into the smallest pores was discarded based on the fact that a reduced enthalpy was measured for the bulk (excess liquid) melting peak. In fact, the calculation of the pore volume for cis-decalin and cyclohexane in the $40 \AA$ pores (from the measurement of the $\Delta H_{f}$ of the excess liquid) gave similar results to the other compounds studied. Annealing at low temperature for $3-4 \mathrm{~h}$ also did not change the result. This suggests that these liquids may be stable in a highly undercooled state in the $40 \AA$ pores, although a greater than $1 / d$ dependence at this small size may also explain the behavior. We also note that no evidence was obtained which suggests vitrification of these two systems.

\section{Effect of pore diameter on $\Delta H_{f}$}

The effect of pore diameter on the measured value of $\Delta H_{f}$ at small pore sizes is dramatic, as shown in Fig. 5. The bulk value is plotted at a diameter of $1000 \AA$, for reference. The observed ratio of $\Delta H_{f}$ (pore) $/ \Delta H_{f}$ (bulk) at the $40 \AA$ pore diameter ranges from 0.28 for heptane to 0.57 for naphthalene. This may be the reason a pore melting endotherm is not detected in cis-decalin and cyclohexane in the $40 \AA$ pores. In the $85 \AA$ pores, these materials show low ratios of $\Delta H_{f}$ (pore) $/ \Delta H_{f}$ (bulk) of $\sim 0.5$, compared to $0.65-0.88$ for the other materials. Although there is some error in the calculated pore melting enthalpy due to the breadth of the peak at small pore size, the error is not large 


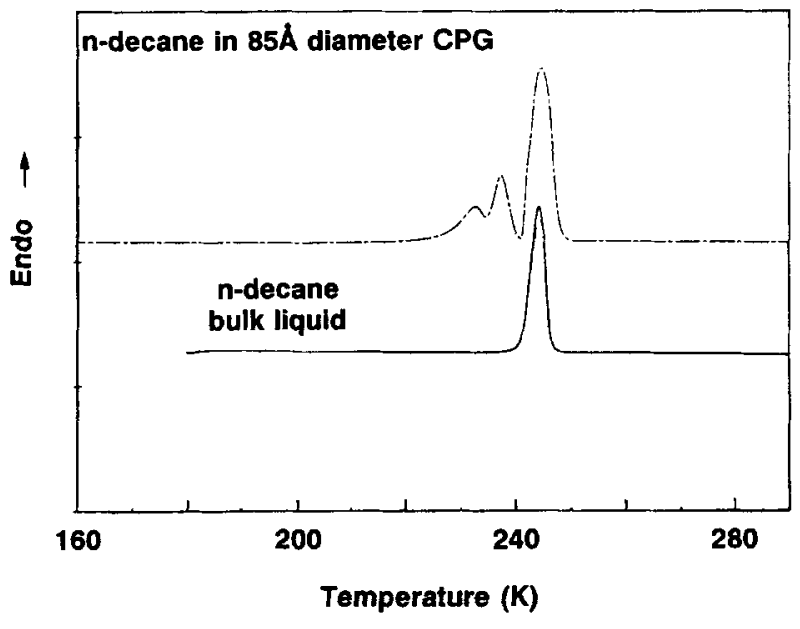

FIG. 6. Anomalous melting behavior of $n$-decane: DSC heating curves of $n$-decane in $85 \AA \mathrm{CPG}$ (heating rate $5 \mathrm{~K} / \mathrm{min}$ ). A scan of pure decane is shown for reference, see the text for details.

enough to account for such a large reduction in $\Delta H_{f} \mathrm{~A}$ small correction for the temperature dependence of $\Delta H_{f}$ is also not enough to account for the large reductions in $\Delta H_{f}$ at the smallest pore sizes. ${ }^{7}$

Many explanations for the reduction in the bulk $\Delta H_{f}$ in the small pores are possible, from both an experimental and theoretical viewpoint. Experimentally, if a liquid layer remains unfrozen on the surface of the glass, all of the material cannot be expected to contribute to the crystalline melting endotherm. If this were the case, however, it is surprising that the proportionality between $\Delta T_{m}$ and $1 / d$ continues at small pore diameter. Related to this is the possible smearing out of the liquid-solid transition as the pore size decreases, where the interface between liquid and solid is large. From a theoretical standpoint, it may be that at very small size, the surface to volume ratio of a crystal becomes important and the bulk properties are affected.

\section{Anomalous melting behavior of some compounds}

All of the data discussed thus far for the pore melting of various organic compounds were well behaved and appeared essentially as shown in Fig. 3 for the example of benzene. For some other compounds this was not the case, and may be an important clue to the behavior of certain materials in confined geometries. In the selection of compounds for this work, we attempted to include a series of $n$-alkanes to complement the data on ring systems. Heptane $(n=7)$ was the first member of this series, since the melting points of the lower alkanes were below the temperature limit of $140 \mathrm{~K}$ for our DSC. Heptane behaved similarly to benzene, described above. The next $n$-alkane studied, $n$-decane $(n=10)$, gave a bimodal pore melting peak shown in Fig. 6, for the $85 \AA$ pores. This was not due to any impurity in the decane, since the pure material gave a single sharp melting endotherm.

Although we do not know the origin of this behavior, diffraction studies on the crystal structure of the pore solid may prove interesting. Neutron diffraction has recently been shown to be a viable technique to study the structure of deuterium oxide ${ }^{34}$ and oxygen ${ }^{35}$ solidified in porous glasses, a rather surprising result considering the small scale of the pores. This suggests that the crystallite size parallel to the cylindrical pore is sufficiently large to yield distinct diffraction peaks and useful information about the effect of confining geometry on crystal structure in the pores. For some $n$-alkanes, multiple solid crystal forms have been reported ${ }^{36}$ and may be relevant to the anomalous melting behavior observed.

\section{ANALYSIS}

In our study of the melting temperature of small organic crystals formed in the pores of CPGs, we analyze the data based on the prediction of a $1 / d$ dependence of $\Delta T_{m}$ according to Eq. (1), and calculate a value of $\sigma_{\mathrm{sl}}$ from the slope of the plot of $\Delta T_{m}$ vs $1 / d$ (Fig. 4). The necessary assumptions are the same as those adopted previously by Gibbs, namely that the values of $\sigma_{\mathrm{sl}}, \Delta H_{f}$ and $\rho_{s}$ are independent of pore size (or crystallite size) and are represented by the bulk values. In addition, it is assumed that the pore diameter is equal to the crystal diameter, although this is actually an upper limit on the crystal diameter. The implicit assumption is that the crystal structure or unit cell of the pore solid is the same as the bulk material. We are aware that a number of other factors might be important, such as surface roughness, transport between the pores and the bulk, and the possibility of a liquid monolayer on the pore surface which might reduce the measured value of $\Delta H_{f}$ and alter the effective pore diameter. In addition, if growing a crystal in a small pore results in a different crystal structure, all the thermodynamic parameters would change.

The values of $\sigma_{\mathrm{sl}}$ calculated from the slopes are given in the first column of Table IV. The first entries were calculated without the smallest pore data, which showed the largest reduction in $\Delta H_{f}$ and are most likely to deviate from the equation due to the small scale. The values calculated including the small pore data are given in parentheses. (Because we have been unable to find values for the solid densities of cis-decalin and trans-decalin, they were calculated by assuming the solid densities are 1.18 times the liquid density; ${ }^{27}$ the solid densities of the other materials were found in the literature. ${ }^{37}$ ) Also given in Table IV are values of $\sigma_{\mathrm{sl}}$ calculated from empirical relations, ${ }^{38-40}$ or literature values reported from different methods of measurement, when available. The empirical relation used was given by Dunning ${ }^{38}$ based on experimental data of homogeneous nucleation experiments from the melt; where the molar ratio of $\sigma_{\mathrm{s} l} / \Delta H_{f}$ per unit area was approximately 0.33 for a number of organics. This was near the lower limit expected for this ratio. Turnbul ${ }^{39}$ gives an empirical estimate of this range as $0.32-0.45$ and theoretical calculations ${ }^{40}$ have predicted a value of 0.45 .

We first compare the empirically calculated values of $\sigma_{\mathrm{si}}$ to our values calculated from the experimental data. In general, the experimentally calculated values from the depression of the melting point (DMP) method used in this 
TABLE IV. Comparison of experimental, empirically calculated, and literature values of $\sigma_{\mathrm{sl}}\left(\mathrm{erg} / \mathrm{cm}^{2}\right)$.

\begin{tabular}{|c|c|c|c|c|c|c|}
\hline \multirow{3}{*}{$\frac{\text { Substance }}{\text { Benzene }}$} & \multirow{2}{*}{\multicolumn{2}{|c|}{$\begin{array}{l}\text { Experimental calculation } \\
\text { from slope of Eq. (1) }\end{array}$}} & \multirow{3}{*}{$\begin{array}{c}\begin{array}{c}\text { Empirical } \\
\text { calculation }^{\mathrm{b}}\end{array} \\
21.5\end{array}$} & \multicolumn{3}{|c|}{ Literature values } \\
\hline & & & & \multirow{2}{*}{$\frac{\sigma_{\mathrm{sl}}}{44 \pm 10}$} & \multirow{2}{*}{$\frac{\text { Method }^{\mathrm{c}}}{\text { GBG }}$} & \multirow{2}{*}{$\frac{\text { Reference }}{41}$} \\
\hline & 15.7 & $(12.9)$ & & & & \\
\hline & & & & $22 \pm 2$ & C & 41 \\
\hline & & & & 19.8 & HN & 41 \\
\hline & & & & 21.4 & HN & 38 \\
\hline Chlorobenzene & 14.1 & $(13.3)$ & 18.4 & & & \\
\hline Cyclohexane & 4.6 & $\ldots$ & 4.8 & & & \\
\hline Heptane & 17.1 & (13.5) & 23.3 & & & \\
\hline \multirow[t]{3}{*}{ Naphthalene } & 8.2 & $(6.1)$ & 31.7 & $61 \pm 11$ & . GBG & 41 \\
\hline & & & & 27.2 & $\mathbf{H N}$ & 41 \\
\hline & & & & 30.1 & HN & 38 \\
\hline cis-decalin & 11.6 & $\ldots$ & 14.4 & & & \\
\hline trans-decalin & 18.4 & $(16.2)$ & 21.4 & & & \\
\hline
\end{tabular}

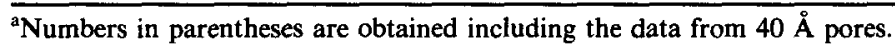

bCalculated from the empirical relation: $\sigma_{\mathrm{sl}, M} / \Delta H_{f, M}$ (Refs. 38 and 39).

${ }^{\circ} \mathrm{GBG}=$ grain boundary groove; $\mathrm{C}=$ conical capillary; and $\mathrm{HN}=$ homogeneous nucleation.

work are $\sim 25 \%$ lower than the empirically calculated values (Table IV). Naphthalene is the notable exception, where a $\sim 75 \%$ lower value of $\sigma_{\mathrm{s} 1}$ was obtained. The low values cannot be easily explained based on the direct application of Eq. (1) to the data obtained in this study. For example, if the lowered $\Delta H_{f}$ observed in the small pores is taken into account, the calculated value of $\sigma_{\mathrm{s} 1}$ would be further reduced. Similarly, reduced values of the bulk $\rho_{s}$, due to poor packing, would also lower $\sigma_{\mathrm{sl}}$. The failure of the assumption that the pore diameter is equal to the crystal diameter, also does not account for the small value of $\sigma_{\mathrm{s} \text { l. }}$. If a few monolayers of liquid on the surface of the pore do not freeze, the effective pore diameter would be smaller and inclusion of such a correction in Eq. (1) would further reduce the calculated value of $\sigma_{\mathrm{sl}}$ One factor which is not explicitly given in Eq. (1) is the equilibrium contact angle between the solid-liquid and solid-substrate interfaces, which is important for freezing studies and determines whether the solid nucleus forms on the substrate (wall) or in the bulk. ${ }^{14}$ We have assumed that the contact angle is $180^{\circ}$ to obtain Eq. (1), as discussed in the Introduction, which implies that the solid is separated from the substrate by a layer of liquid. If the contact angle is actually somewhat less than $180^{\circ}$, this would increase the calculated value of $\sigma_{\mathrm{sl}}$. We note that the especially low value of $\sigma_{\mathrm{sl}}$ obtained for naphthalene may be due to the large anisotropy reported for $\sigma_{s l}$ of this compound $(20 \%) .{ }^{41}$ It is also possible that changes in the crystallographic form occur when naphthalene is crystallized in a constrained geometry, and may be an interesting subject of future study.

A comparison of the values of $\sigma_{\mathrm{sl}}$ calculated here with those obtained by other experimental methods is next considered. Our values are generally comparable to values obtained by the methods of homogeneous nucleation (HN) and conical capillary (C), but low compared to the grain boundary groove (CBG) method, within the large variability of such measurements reported in the literature. The inherent difficulties of determining reliable values of $\sigma_{\text {sl }}$ have been reviewed by Bilgram, ${ }^{21}$ Jones, ${ }^{41}$ and Woodruff. ${ }^{42}$ The most common methods are $\mathrm{HN}$ and GBG experiments. In the case of $\mathrm{HN}$, widely used for both metals and organics, the most serious questions arise from the uncertainty of applying macroscopic thermodynamics to systems as small as the solid nucleus (typically $10 \AA$ in size), the question of trace impurities which would result in heterogeneous rather than homogeneous nucleation, and the fact that in a nucleation experiment, $\sigma_{\mathrm{sl}}$ is determined as much as $200 \mathrm{~K}$ below $T_{m}$ without consideration of a temperature coefficient for $\sigma_{\mathrm{sl}}{ }^{41}$ The GBG method is also called the method of interface intersections, and the measured angles of intersection can be used to derive a value of $\sigma_{\mathrm{sl}}$. The GBG method is very difficult to apply to pure materials that are opaque, such as metals, and has more widespread use in the study of metal alloys. ${ }^{42}$ The conical capillary (C) method was one of the earliest to measure $\sigma_{\mathrm{sl}}$ from the direct application of the Gibbs-Thomson equation, but has not been used subsequently. This is due to practical disadvantages, such as the extremely good temperature control required and lack of suitability for impure systems.

The method of obtaining $\sigma_{\mathrm{sl}}$ from measurements of DMP of small crystals used in this work is similar to HN in size scale and may suffer criticisms in this regard, although trace impurities and temperature dependence of $\sigma_{\mathrm{sl}}$ should be much less important. As mentioned earlier, narrow pore size distribution materials of sufficiently small pore diameters have become readily available in recent years, and the method of DMP should be reconsidered as a method of determining $\sigma_{\mathrm{sl}}$. It may be at least as reliable and easier to use than the $\mathrm{HN}$ method. This has also been noted by the workers employing thermoporimetry. ${ }^{30}$

Some mention should be made to the possible relation of this work to cluster theory, ${ }^{43}$ for although the geometry is different, the size scale is similar. The melting point of microcrystals, or clusters, should clearly demonstrate the manner in which the properties approach those of the bulk phase as the size of the particles increase, although more work in this area is needed. ${ }^{44}$ Computer simulations indicate that the melting transition of Lennard-Jones microcrystals confined to spherical cavities is related to specific 
geometrical factors. The Lennard-Jones clusters are predicted to have a melting temperature that is directly related to the number of atoms in the cluster. The equation for the melting point depression $\Delta T_{m}^{*}$ (where $*$ denotes quantities reduced by the Lennard-Jones parameters) is

$$
\Delta T_{m}^{*}=A N^{-1 / 3}-B N^{-2 / 3},
$$

where $A$ and $B$ are unknown constants and $N$ is the number of atoms in the cluster. This equation is derived for spherical particles so the assumption, $R_{s} \propto N^{-1 / 3}$ is made, and the leading term in Eq. (2) has the same inverse radius dependence as predicted by Eq. (1). However, at very small radius (small $N$ ) the $N^{-2 / 3}$ term becomes significant and Eq. (2) predicts a dependence of $\Delta T_{m}$ which differs from $1 / d$. Rather it predicts that $\Delta T_{m} \propto 1 / d^{2}$. This would not be supported from our data, which include sizes to 40 $\AA$. Alternatively, that continuum equations apply to both organics and metals ${ }^{18}$ at the size scales studied implies that bulk properties are still valid. This is in accord with the work $^{45}$ of Lessen, Asher and Brucat on vanadium argon clusters with $n$ atoms, $\mathrm{VAr}_{n}^{+}$, where bulk behavior is approached with just $n=7$. One may object that the large reduction in $\Delta H_{f}$ reported in this work is counter to the argument of bulk behavior, but at this time we do not know whether the reduction of $\Delta H_{f}$ is due to the diffuse nature of the solid-liquid interface, the presence of a liquid layer, or an actual reduction in the enthalpy of fusion of a very small crystal. Further experimental work using techniques such as scattering and nuclear magnetic resonance might help resolve these questions.

\section{SUMMARY}

Studies on the effect of finite size on the bulk properties of materials confined to very small pores is a fundamental bridge of understanding between the molecular scale and its relationship to the bulk scale. In this work, we have studied the crystalline melting transition and melting enthalpy of a series of organic solids confined to small pores, and determined the effect of pore diameter on the magnitude of the melting point depression and the apparent enthalpy of fusion $\Delta H_{f}$. The porous media used in this work were controlled pore glasses with well characterized and narrow pore size distributions, and pore diameters in the range of $40-730 \AA$. These unique materials provide a carefully controlled environment in which to grow small organic crystals of specific diameters.

The melting point depression $\Delta T_{m}$ was found to be an inverse linear function of the crystal diameter $d$ in accordance with the continuum theory of Eq. (1). A few earlier studies on $\Delta T_{m}$ as a function of pore diameter for small inorganic molecules such as water ${ }^{11}$ and oxygen ${ }^{5}$ have been previously reported to have similar $\Delta T_{m}$ vs $1 / d$ dependences, but in general, controlled studies on liquids confined in pores of different diameter are rare. Quantitative application of Eq. (1) gave values of the solid-liquid interfacial energy $\sigma_{\mathrm{s}}$, which are similar to, but somewhat lower than, those obtained by homogeneous nucleation methods. This may be due to the unknown factor of the contact angle at the solid-liquid interface. Naphthalene gave an anomalously low value by this method and possible explanations, such as anisotropy of $\sigma_{\mathrm{sl}}$, were considered.

A unique feature of this work is the quantitative measure of the enthalpy of fusion $\Delta H_{f}$ of the solid in the pores as a function of pore diameter. The values of $\Delta H_{f}$ were found to be a function of pore diameter and greatly reduced $(40-70 \%)$ in the smallest pores. For some compounds (cis-decalin and cyclohexane) no melting endotherm was detected in the $40 \AA$ pores even well below the expected melting temperature. Possible explanations for this behavior were offered, such as the low enthalpy of fusion of these two materials (relative to the other materials studied) and the possible stability of the undercooled liquid in the confined geometry of the pores. Relevant to this may be studies on the liquid-glass transition of organic liquids confined in small pores, which we report in a set of related experiments. ${ }^{46}$

This work is part of an ongoing study of the freezing and melting behavior of organic solvents in thermoreversible $^{47}$ and cross-linked ${ }^{48,49}$ polymer gels. Under certain conditions, the mesh-like, network structure of polymer chains forces the solvent to stay "compartmentalized," 13 so that upon freezing, only very small solvent crystals can form. The melting behavior then may mimic that observed in CPG in some regards, and we hope to relate the data of these two studies.

\section{ACKNOWLEDGMENTS}

The authors are indebted to Dr. Wolfgang Haller of NIST for providing the samples of controlled pore glass and instruction on how to best derivatize the surfaces. We would also like to thank Dr. Jack Douglas of NIST for his many helpful comments on the manuscript. The lowtemperature modification of the DSC was made by Willard Roberts of the Center for Building Technology at NIST, and use of that equipment is gratefully acknowledged.

'S. Brunauer, The Adsorption of Gases and Vapors, Vol. I, Physical Adsorption (Princeton University, Princeton, 1943), p. 444.

${ }^{2}$ W. A. Patrick and W. A. Kemper, J. Phys. Chem. 42, 369 (1938).

${ }^{3}$ D. D. Awschalom and J. Warnock, in Molecular Dynamics in Restricted Geometries, edited by J. Klafter and J. M. Drake (Wiley, New York, 1989), Chap. 12.

${ }^{4}$ G. G. Litvan, Can. J. Chem. 44, 2617 (1966)

${ }^{5}$ D. D. Awschalom and J. Warnock, Phys. Rev. B 35, 6774 (1987).

${ }^{6}$ R. Defay, I. Prigogine, A. Bellemans, and D. H. Everett, Surface Tension and Adsorption (Wiley, New York, 1966).

${ }^{7}$ B. R. Puri, D. D. Singh, and Y. P. Myer, Trans. Faraday Soc. 53, 530 (1957).

${ }^{8}$ J. L. Tell and H. J. Maris, Phy. Rev. B 28, 5122 (1983).

${ }^{9}$ B. V. Enustun, H. S. Senturk, and O. Yurdakul, J. Coll. Int. Sci. 65, 509 (1978).

${ }^{10}$ C. Hodgson and R. McIntosh, Can. J. Chem. 38, 958 (1960).

${ }^{11}$ G. K. Rennie and J. Clifford, J. Chem. Soc. F1 73, 680 (1977).

${ }^{12}$ K. Shirahama, M. Kubota, S. Ogawa, N. Wada, and T. Watanabe, Phys. Rev. Lett. 64, 1541 (1990).

${ }^{13} \mathrm{~N}$. Murase, K. Gonda, and T. Watanabe, J. Phys. Chem. 90, 5420 (1986), and reference cited therein.

${ }^{14}$ K. A. Jackson and B. Chalmers, J. Appl. Phys. 29, 1178 (1958)

${ }^{15}$ R. F. Feldman and H. Cheng-yi, Cement Concrete Res. 15, 765 (1985).

${ }^{16}$ W. Haller, Nature 206, 693 (1965).

${ }^{17} \mathrm{Ph}$. Buffat and J-P. Borel, Phys. Rev. A 13, 2287 (1976).

${ }^{18}$ P. R. Couchman and W. A. Jesser, Nature 269, 481 (1977). 
${ }^{19}$ F. Ehrburger and J. Lahage, Colloids Surf. 23, 105 (1987).

${ }^{20}$ J. D. Hoffman, G. T. Davis, and J. I. Lauritzen, in Treatise on SolidState Chemistry, edited by N. B. Hannay (Plenum, New York, 1976).

${ }^{21}$ J. H. Bilgram, Phys. Rep. 153, 1 (1987).

${ }^{22}$ J. W. Gibbs, Collected Works (New York, 1928).

${ }^{23}$ W. Thomson (Lord Kelvin), Philos. Mag. 42, 448 (1871)

${ }^{24}$ Certain commercial companies are named in order to specify adequately the experimental procedure. This in no way implies endorsement or recommendation by NIST.

${ }^{25}$ W. Haller, in Solid Phase Biochemistry, edited by W. H. Scouten (Wiley, New York, 1983), Ch. 11.

${ }^{26}$ B. W. Sands, Y. S. Kim, and J. L. Bass, J. Chrom. 360, 353 (1986).

${ }^{27}$ Handbook of Chemistry and Physics, 56th ed. edited by R. C. Weast (Chemical Rubber Co., Cleveland, 1975).

${ }^{28}$ J. P. McCullough, H. L. Finke, J. F. Messerly, S. S. Todd, T. C. Kinchelae, and G. Waddington, J. Chem Phys. 61, 1105 (1957).

${ }^{29}$ S. S. Chang and A. B. Bestul, J. Chem. Phys, 56, 503 (1972).

${ }^{30}$ L. G. Homshaw, J. Coll. Int. Sci. 84, 141 (1981).

${ }^{31}$ M. Brun, A. Lallemand, J.-F. Quinson, and C. Eyraud, Thermochim. Acta 21, 59 (1977).

${ }^{32} \mathrm{~J}-\mathrm{F}$. Quinson, M. Brun, R. Spitz, and M. Bartholin, Makromol. Chem. 185, 1105 (1984).

${ }^{33}$ The cyclohexane lower limit is $\sim 190 \mathrm{~K}$ because the pore melting peak was not observed below the solid-solid phase transition observed for this compound at $186 \mathrm{~K}$ [R. Kahn, R. Fourme, D. Andre, and M. Renaud, Acta Cryst. B 29, 131 (1973)].
${ }^{34}$ M. Dunn, J. C. Dore, and P. Chieux, J. Cryst. Growth 92, 233 (1988).

${ }^{35}$ P. E. Sokol, K. W. Herwig, and M. Pang, Bull. APS 35, 442 (1990).

${ }^{36}$ E. F. Westrum and J. P. McCullough, in Physics and Chemistry of the Organic Solid State, edited by D. Fox et al. (Interscience, New York, 1963), Chap. 1.

${ }^{37}$ Crystal Data Determinative Tables, 3rd. ed., edited by J. D. H. Donnay and H. M. Ondik (U. S. Department of Commerce (NBS) and JCPDS, Washington, D. C., 1972), Vol. 1.

${ }^{38}$ W. J. Dunning, in Physics and Chemistry of the Organic Solid State, edited by D. Fox et al. (Interscience, New York, 1963), Chap. 7.

${ }^{39}$ D. Turnbull, J. Appl. Phys. 21, 1022 (1950).

${ }^{40}$ J. Q. Broughton and G. H. Gilmer, J. Chem. Phys. 84, 5759 (1986).

${ }^{41}$ D. R. H. Jones, J. Mater. Sci. 9, 1 (1974).

${ }^{42}$ D. P. Woodruff, The Solid-Liquid Interface (Cambridge University, Oxford, 1973), Chap. 2.

${ }^{43}$ N. Quirke, Mol. Simul. 1, 249 (1988).

${ }^{44}$ D. J. Wales and R. S. Berry, J. Chem. Phys. 92, 4473 (1990).

${ }^{45}$ D. Lessen, R. L. Asher, and P. J. Brucat, Int. J. Mass Spectrosc. Ion Process. (in press).

${ }^{46}$ C. L. Jackson and G. B. McKenna, J. Non-Cryst. Solids (in press).

${ }^{47}$ C. L. Jackson and G. B. McKenna, Am. Chem. Soc.: Polym. Preprints 31(2), 607 (1990).

${ }^{48}$ C. L. Jackson and G. B. McKenna, Rubber Chem. Tech. (in press).

${ }^{49}$ B. B. Boonstra, F. A. Heckman, and G. L. Taylor, J. Appl. Polym. Sci. 12, 223 (1968). 